Artigo / Article

\title{
O papel da terapia de manutenção no Mieloma Múltiplo
}

The role of maintenance therapy for Multiple Myeloma

Rosane Bittencourt

"O papel da terapia de manutenção no MM é controverso."

Berenson

Os atuais estudos sobre a biologia e o comportamento da célula do mieloma múltiplo (MM) fazem com que os especialistas mudem de postura frente as possibilidades terapêuticas, fixando como alvos o prolongamento da remissão e sobrevida, passando a olhar essa doença maligna fatal como uma doença de curso crônico.

A introdução da quimioterapia de altas doses seguida pelo transplante autólogo iniciou uma nova era para o MM, ampliando a sobrevida para além dos sessenta meses. Neste contexto, inicia-se a cruzada para manter em tempo máximo esta taxa de resposta obtida, bem como para controlar a doença residual mínima.

O conceito de terapia de manutenção passou a ser uma estratégia viável, considerando o advento de novas drogas ativas e eficazes no controle da doença e na terapia de suporte. $\mathrm{O}$ primeiro agente estudado com a finalidade de manter a resposta provocada pela terapia inicial foi o interferon- $\alpha$ (IFN- $\alpha) .{ }^{1}$ Durante anos, vários ensaios clínicos defenderam o uso do IFN- $\alpha$ ou a associação entre o IFN- $\alpha$ e corticóides na expectativa de mudar as sobrevidas livre de mieloma e global. Ensaios com corticóides, seguidos dos recentes estudos com agentes antiangiogênicos, como a talidomida e seus análogos, o bortozomibe e até mesmo vacinas, são

Hematologista. Coordenadora dos Programas de Mieloma Múltiplo e Transplante Autólogo do Serviço de Hematologia do Hospital de Clínicas de Porto Alegre - RS.

Correspondência: Rosane Bittencourt

Rua Jaime Telles, 133/501 - Petrópolis

90460-030 - Porto Alegre-RS - Brasil

Tel.: (51) 2101-8317

E-mail: rbittencourt@hcpa.ufrgs.br 
cogitados com a finalidade de prolongar a fase de estabilidade da doença, aumentar a sobrevida e transformar a história do MM.

\section{Interferon}

O primeiro trabalho que utilizou o interferon- $\alpha$ (IFN- $\alpha)$, na terapia de mieloma múltiplo foi publicado em 1979. ${ }^{2}$ Neste estudo, $3 \mathrm{mU}$ de IFN- $\alpha$ aplicadas intramuscular, diariamente, foram empregadas como terapia de indução, mostrando que 30\% dos pacientes obtinham respostas duradouras entre 319 meses. Estes resultados clínicos levaram os investigadores a olhar este modificador biológico como uma terapia em potencial.

A partir do estudo de Mandelli, iniciado em 1985 e publicado em 1990, o IFN- $\alpha$ foi cogitado como droga de manutenção da fase de estabilização do MM (plateau). Passou a ser recomendado após a terapia de indução ou transplante autólogo de células-tronco hematopoéticas. ${ }^{1}$ O IFN- $\alpha$, usado como agente único nas doses de $3-5 \mathrm{mU} / \mathrm{m}^{2}$, subcutâneo (SC) três vezes na semana, é o regime de manutenção mais antigo e mais analisado. No estudo italiano, 101 pacientes respondedores à terapia inicial foram randomizados: 50 para manutenção com IFN- $\alpha$ e 51 sem terapia. As médias de duração de resposta e sobrevida global foram respectivamente 26 meses (m) e $52 \mathrm{~m}$ usando o IFN- $\alpha$ contra $14 \mathrm{~m}$ e $39 \mathrm{~m}$ no grupo sem manutenção. A conclusão deste estudo foi que o IFN- $\alpha$ prolonga o tempo de resposta e a sobrevida global, porém não foi isento de efeitos indesejáveis como síndrome flu-like, febre e mialgias.

Seguindo Mandelli, um grande número de estudos foi iniciado analisando o potencial do IFN- $\alpha$ em prolongar fases livres da doença e a sobrevida global. ${ }^{3-8}$ Os resultados foram comuns entre os estudos, evidenciando a tendência de uma maior fase de inatividade do mieloma, mas em contrapartida ressaltando as toxicidades do IFN- $\alpha$. Finalizam suas impressões recomendando metanálises para avaliar o poder da manutenção com o IFN- $\alpha$ na sobrevida dos pacientes. ${ }^{6}$

Entusiasmados pelos resultados divulgados pelo grupo italiano em 1990, surgiram na literatura mais cinco grandes estudos randomizados comparando o tratamento de manutenção com interferon com os grupos controles não tratados: dois destes demonstraram clara vantagem na duração da resposta e na sobrevida, ${ }^{3,9}$ um apenas comprovou melhora na duração de resposta sem impacto na sobrevida, ${ }^{4}$ enquanto os dois últimos não atingiram melhoras significativas na duração da resposta ou na sobrevida. ${ }^{10,11}$ No estudo de Browman e colaboradores ${ }^{3}$ há registros a favor da manutenção: uma sobrevida média de 44 meses pró manutenção contra os 33 meses sem manutenção; já no estudo de Salmon, os pacientes randomizados ao esquema manutenção IFN- $\alpha$ não mostraram vantagens na sobrevida livre de doença ou na sobrevida global, além de relatos de toxicidade associada. ${ }^{11}$
Alguns estudos, como o conduzido pelo Medical Research Council (MRC), salientam que não há diferenças significativas entre o grupo sem terapia e o grupo mantido com IFN- $\alpha$ após a terapia indução, salientado que a recaída após a manutenção com IFN- $\alpha$ foi mais intensa, menos responsiva à terapia de resgate e com encurtamento da sobrevida. ${ }^{6,12}$

Passados quase vinte anos da publicação de Mandelli, o seguimento dos pacientes em tratamento de manutenção com IFN- $\alpha$ permitiu as várias observações descritas. Inicialmente, o estudo randomizado conduzido pelo NMSG evidenciou um ganho de seis meses em sobrevida livre de doença, porém apenas um ganho não significativo na sobrevida global, além de relatar a redução da qualidade de vida durante o período de uso do IFN- $\alpha{ }^{13} \mathrm{Em}$ outra metanálise, 1.543 pacientes participando de 12 ensaios clínicos foram randomizados para receber IFN- $\alpha$ após terapia de indução, enquanto 2.469 pacientes foram randomizados a iniciar o IFN- $\alpha$ desde a indução, permanecendo em uso posteriormente como manutenção. Os primeiros resultados da terapia de manutenção pareciam otimistas, demonstrando ganhos de seis e sete meses nas sobrevidas livre de doença e global respectivamente em comparação aos pacientes que não receberam manutenção; entretanto, analisando os dois grupos conjuntamente, estes ganhos na sobrevida global resumiam-se em apenas quatro meses. Os resultados positivos a favor da manutenção com o IFN- $\alpha$ foram desbancados após estudos de metanálises. ${ }^{14}$ Análises preliminares do US Intergroup demonstraram ausência de benefícios na prática da manutenção com o IFN- $\alpha$, além de apresentar parefeitos. Concluem que a magnitude do benefício do IFN- $\alpha$ é pequena. ${ }^{15,16}$

Um estudo aberto fase III reporta o resultado da randomização para manutenção IFN- $\alpha$ após indução. Noventa pacientes que alcançaram remissão com o melfalano e prednisona foram randomizados entre IFN- $\alpha$ e não manutenção. Os pacientes foram acompanhados durante 97 meses, o uso do IFN- $\alpha$ foi associado com melhora na sobrevida livre de mieloma (13,5 m x 8,4 m), mas não houve melhora na sobrevida global ( $40 \mathrm{~m}$ x $38 \mathrm{~m}$ ). Em 1/3 dos pacientes a terapia foi descontinuada devida à toxicidade do IFN- $\alpha .{ }^{17}$ Outros estudos não randomizados referem uma pobre complacência ao IFN- $\alpha$ pelos efeitos sistêmicos e hematológicos. ${ }^{18,19,20}$

A maioria dos estudos randomizados preconizando a manutenção com IFN- $\alpha$ demonstra significativa melhora na sobrevida livre de doença, porém a ação na sobrevida global permanece sem benefício claro. Conseqüentemente, fora de estudos clínicos, a terapia de manutenção com IFN- $\alpha$ não tem evidências suficientes para ser recomendada.

\section{Corticóides}

A capacidade de induzir apoptose na linhagem hematopoética linfóide torna atraente a inclusão dos 
glicocorticóides nas linhas terapêuticas para doenças linfoproliferativas como o MM. ${ }^{21}$

A estratégia de manutenção com o IFN- $\alpha$, após a terapia de indução ou das altas doses resgatadas pelo transplante, abriu espaço para que outros medicamentos fossem testados com este fim.

A década de 90 deu início a uma série de publicações enfocando a dexametasona ou a prednisona como opções de manutenção. Palumbo e colaboradores testaram a associação entre IFN- $\alpha$ e os corticóides após a quimioterapia convencional de indução num pequeno grupo de pacientes. Seus achados foram que a manutenção com IFN- $\alpha$ + corticóides reduzia a massa tumoral residual e poderia prolongar o tempo de remissão. ${ }^{22}$

Estudos randomizados entre o IFN- $\alpha$ e a dexametasona mostraram atividade semelhante entre essas duas opções de manutenção. Os efeitos colaterais foram toleráveis para ambos, e nenhum dos esquemas foi consistente para alterar a sobrevida global. ${ }^{23}$ No Canadian NCIC trial houve superioridade no tempo de sobrevida livre de doença com pulsos de dexametasona $40 \mathrm{mg} /$ dia durante quatro dias no mês, mas também falhou em causar impacto na sobrevida global, além de desencadear efeitos colaterais indesejáveis como níveis tensionais elevados, hiperglicemia, hipertricose e obesidade. ${ }^{24}$

Berenson e colaboradore ${ }^{25}$ reportaram o prolongamento tanto da sobrevida livre de doença, quanto da sobrevida global nos pacientes que receberam terapia de manutenção com prednisona $50 \mathrm{mg}$ em dias alternados. Este estudo foi passível de muitas críticas pelo seu desenho e forma de seleção dos pacientes incluídos.

Um maior número de estudos associa o corticóide ao IFN- $\alpha$, ou a agentes antiangiogênicos otimizando a sobrevida livre de recaída. ${ }^{26,27}$ No estudo publicado pelo Salmon, o grupo randomizado para manutenção com IFN- $\alpha$ + prednisona alcançou 57 meses de sobrevida livre de progressão contra 46 meses daqueles que receberam somente o IFN- $\alpha$. Os resultados corroboram com a maioria das demais revisões: enfatizam a vantagem na sobrevida livre de evento, alertam para efeitos colaterais dos agentes, mas não mostram resultados capazes de mudar significativamente o tempo de sobrevida global.

As publicações enfocando a corticoterapia como terapia de manutenção, após a obtenção de resposta completa, não são suficientemente consistentes para sua incorporação nas diretrizes de tratamento.

\section{Talidomida}

A talidomida provou causar impacto sobre as discrasias plasmocitárias. Através de suas atividades antiangiogênicas, imunomodulatórias e promotora de apoptose é um agente muito ativo no combate ao MM. ${ }^{28}$ Este alto grau de atividade na doença recaída ou refratária fez com que os pesquisadores lançassem a proposta de usar a talidomida como terapia de manutenção, basicamente após o transplante autólogo de células-tronco hematopoéticas.

A introdução da talidomida no rol das opções para manutenção ainda é um fenômeno recente. As primeiras impressões sobre os efeitos da talidomida na manutenção começaram a ser divulgadas a partir de 2003 em abstracts do ASH posteriormente publicados. ${ }^{29,30}$ Os resultados iniciais favoreciam o prolongamento de sobrevida livre de mieloma, mas sem tempo suficiente para avaliar a sobrevida global. Referiam efeitos colaterais dignos de nota pelo grau da toxicidade exibida: neuropatia, rash, sonolência, tonturas, constipação, impotência e eventos tromboembólicos, ocasionando a interrupção temporária ou definitiva da manutenção para alguns.

Em 2004, Santos e colaboradores reiteraram efeitos favoráveis da talidomida em pacientes submetidos ao transplante autólogo que mantiveram sobrevida livre de doença durante 172 semanas, hora do início da manutenção até análise dos dados. Os efeitos adversos apresentados: constipação, pele seca, rash e zumbidos foram toleráveis por grande parte do grupo, sendo que apenas dois pacientes interromperam seu uso. ${ }^{31}$

Os dados preliminares apresentados pelo grupo francês sugeriam a talidomida como efetiva na manutenção após o transplante autólogo. O estudo do Intergroupe Francophone du Myeloma (IFM 99 02) randomizou os pacientes pós-transplante autólogo em três braços: a)sem manutenção, b)manutenção apenas com pamidronato e c) manutenção associada com pamidronato e talidomida. Os resultados deste estudo mostraram claramente vantagens no uso associado da talidomida ao pamidronato: redução da taxa de progressão da doença e significativo aumento na sobrevida livre de mieloma. ${ }^{32}$ No IFM 99 04, recentemente publicado, Attal e colaboradores confirmam que, após três anos de randomização, a sobrevida livre de doença foi 36\% nos pacientes sem manutenção, $37 \%$ nos que fizeram somente pamidronato e $52 \%$ no grupo da manutenção pamidronato associado a talidomida. Nesta publicação, a probabilidade de sobrevida global em quatro anos foi superior na manutenção com talidomida: $87 \%$ contra os $74 \%$ nos pacientes com pamidronato e $77 \%$ naqueles sem manutenção. ${ }^{33}$ Este é o primeiro estudo randomizado evidenciando impacto na sobrevida global com a manutenção seguida ao transplante autólogo. Porém, o próprio estudo ressalva que o impacto na sobrevida é nítido para o grupo de pacientes que não obteve resposta completa ou quase completa antes da manutenção. Também é interessante observar que aqueles pacientes com deleção de 13 não tiveram impacto na sobrevida global usando a manutenção.

As publicações sobre a terapia de manutenção para o mieloma nestes dois últimos anos (2005 e 2006) seguem advogando o uso da talidomida como alternativa para aumentar a freqüência da resposta completa, estender a sobrevida livre de doença e possivelmente também a sobre- 
vida global em determinado grupo de pacientes. ${ }^{33,34,35}$ Os resultados relacionados ao tempo livre da doença são positivos, mas carregam as desvantagens dos efeitos colaterais incapacitantes como a neuropatia periférica e a trombose venosa profunda.

A eficiência da talidomida em consolidar a resposta e aumentar o tempo livre da doença é reconhecida, entretanto, os benefícios sobre a sobrevida global estão confirmados para o grupo de pacientes que não atingiu uma resposta completa ou resposta muito próxima à completa com a terapia de indução seguida do transplante autólogo.

\section{Inibidores de osteólise}

O acometimento ósseo no mieloma é importante causa de morbi-mortalidade. Os inibidores de osteólise possuem a capacidade de bloquear os osteoclastos, impedindo sua ação osteolítica e desencadeando sua apoptose. ${ }^{36}$ Além da ação específica sobre a atividade osteoclástica, os inibidores de osteólise, tomando como protótipo o pamidronato, possuem uma ação antitumor através da inibição da interleucina-6 (IL-6), fator que coloca este grupo de drogas nas possibilidades de manutenção após a quimioterapia convencional ou após altas doses com MEL 200.

Os estudos reportados na literatura incluem o pamidronato em associação com corticóides e mais freqüentemente com a talidomida como esquemas elegíveis para a manutenção após obtenção de resposta, enfatizando o benefício do efeito antimieloma do inibidor de osteólise. ${ }^{32,33,37}$

A aplicação de pamidronato na manutenção é defendida como uma estratégia efetiva principalmente sobre a doença óssea, mostrando que os pacientes reduzem as dores e apresentam menos eventos ósseos e retardam o tempo de aparecimento de novas lesões. ${ }^{31,38}$

O benefício do pamidronato sobre a doença óssea não suscita dúvidas e está bem recomendado, embora o tempo de uso ainda esteja em discussão. Em termos de impacto na mortalidade do mieloma não houve as mesmas evidências relativas aos eventos esqueléticos, fato que não sustenta sua prática como terapia de manutenção antimieloma.

\section{Vacinas}

Outro tipo de terapia de manutenção estudado há alguns anos são as vacinas, atuantes após a realização de transplante autólogo, com a finalidade de induzir respostas imunes e até a reduzir doença residual mínima. ${ }^{39} \mathrm{O}$ principal tipo de vacina é a específica contra o idiótipo do mieloma, embora as vacinas antiDNA também estejam sendo testadas. ${ }^{40,41}$ Atualmente, o emprego das vacinas está autorizado apenas em ensaios clínicos. Os resultados são preliminares, mas apontam aumento de respostas imunes satisfatórias, embora sem impacto nas sobrevidas livre de doença e global.

\section{Novas terapias}

As novas terapias como o inibidor de proteasoma (bortezomibe) e os análogos da talidomida (lenalidomide) estão despontando como potenciais armas antitumorais capazes de induzir maiores índices de resposta. ${ }^{42}$ Baseado na atividade antimieloma destas drogas, há especulações que uma manutenção com bortozomibe possa intensificar a resposta inicialmente obtida. ${ }^{43}$ Não há ainda nenhum estudo randomizado confirmando esta conduta.

\section{Conclusões}

O papel da terapia de manutenção no mieloma múltiplo é uma das questões mais difíceis para os médicos, até mesmo porque muitos pacientes sentem-se seguros quando estão sob alguma forma de tratamento. Nas últimas décadas, o tema manutenção é constante nas publicações sobre MM, e várias estratégias estão sendo preconizadas, porém poucas apresentando claro benefício para a sobrevida global na maioria dos pacientes. Portanto, ainda não há consenso sobre o papel da manutenção no MM porque seu impacto na mortalidade precisa ser mais bem demonstrado.

O MM é uma doença de padrão heterogêneo, apresentando um comportamento diferente para cada indivíduo. Considerando as características da doença e de cada doente, a recomendação é de individualizar a necessidade da terapia de manutenção. Attal ${ }^{33}$ mostrou que a manutenção tem benefício nas sobrevidas livre de doença e global naqueles pacientes sem resposta quase completa ou sem resposta completa, onde a talidomida como manutenção foi capaz de converter a resposta parcial em resposta completa.

Talvez uma estratégia para definir a manutenção possa ser estabelecida conforme a resposta obtida com a terapia de indução, esquema sugerido pelo quadro abaixo. Havendo a indicação de manutenção, a escolha do agente deve ser feita com base no custo versus benefício, analisando-se a aderência e tolerância ao tratamento; factibilidade de uso; efeitos colaterais e os resultados das metanálises quanto ao impacto nas sobrevidas livre de doença e global.

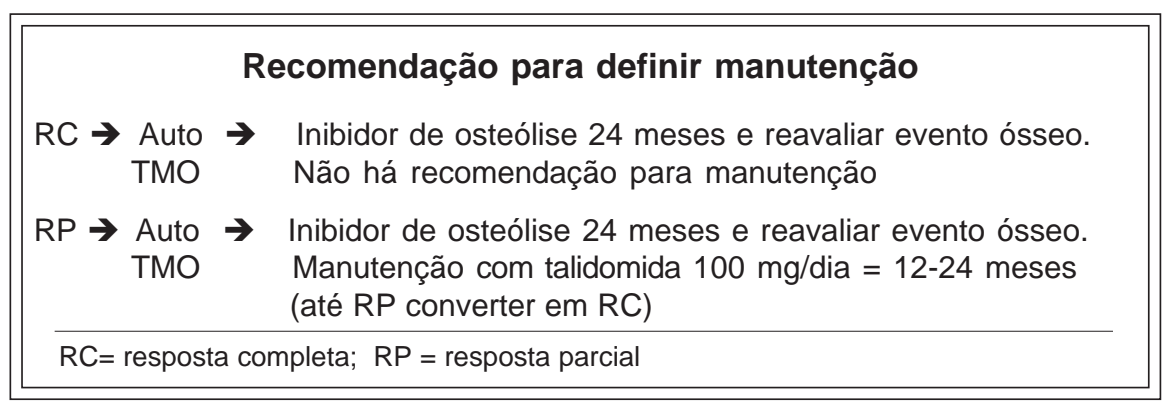




\begin{abstract}
The use of maintenance therapy for patients with multiple myeloma (MM) and complete or almost complete remission is still under discussion. Although not curative, high dose chemotherapy followed by autologous bone marrow transplantation significantly prolongs overall survival time. The addition of maintenance therapy seems to be a valuable alternative aiming at longer inactive disease periods (plateau) or even as an effective therapy for minimal residual disease. It may also bring higher rates of complete response to patients with an initial partial response. Over more than two decades, maintenance therapy for MM has been discussed and proposed. Since then various drugs and their combinations have been studied for this purpose, such as interferon-alpha, glucocorticoids - including dexamethasone and prednisone - and the more recently antiangiogenic drugs including thalidomide and its analogs. Clinical trials with each of these agents have shown advantages and, sometimes, disadvantages in terms of disease-free survival, overall survival and toxicity. A general review of these studies draws attention to the lack of evidence favoring unequivocal benefits in overall survival. In a time when novel and promising therapies arrive we shall emphasize the need of individual therapeutic approaches in terms of maintenance therapy, considering the prognosis and risk factors of each patient. Rev. bras. hematol. hemoter. 2007;29(1):54-59.
\end{abstract}

Key words: Multiple myeloma; complete response; maintenance therapy; interferon; glucocorticosteroids.

\section{Referências Bibliográficas}

1. Mandelli F, Avvisati G, Amadori S, Boccadoro M, Gernone A, Lauta $\mathrm{VM}$, et al. Maintenance treatment ith recombinant interferon alfa$2 \mathrm{~b}$ in patients with multiple myeloma responding to conventional induction chemotherapy. NEJM 1990;322:1.430-1.434.

2. Ahre A, Bjorkholm M, Mellstedt H, et al: Human leukocyte interferon and intermittent high-dose melphalan-predniMone administration in the treatment of multiple myeloma: A randomized clinical trial from the Myeloma Croup of Central Sweden. Cancer Treat Rep 1984;68:1.331.

3. Browman GP, Bergsagel D, Sicheri D, O'Reilley S, Wilsnon KS, Rubin $\mathrm{S}$, et al. Randomized trial of interferon maintenance in multiple myeloma: a study of National Cancer Institute of Canada Clinical Trials Group. Journal Clinical Oncology 1995;13:2.354-2.360.

4. Westin J, Rodjer S, Turesson I, Cortelezzi A, Hjorth M, Zador G. Interferon alfa $2 b$ vs. no maintenance therapy during the plateau phase in multiple myeloma: a randomized study. British Journal of Haematology 1995;89:561-568.

5. Joshua DE, Penny R, Matthews JP, Laidlaw CR, Gibson J, Bradstock $\mathrm{K}$, et al. Australian Leukemia Study Group Myeloma II: a randomized trial of intensive combination chemotherapy with or without interferon in patients with myeloma. British Journal of Haematology 1997;97:38-45.

6. Drayson MT, Chapman CE, Dunn JA, Olujohungbe AB, Maclennam IC. MRC trial of $2 \beta$-interferon maintenance therapy in first plateau phase of multiple myeloma. British Journal of Haematology 1998;101:195-202.

7. Cunningham D, Powles R, Malpas J, Raje N, Milan S, Viner C, et al. A randomized trial of maintenance interferon following high-dose chemotherapy in multiple myeloma: lng-term follow-up results. British Journal of Haematology 1998;102:495-502.
8. Bjorkstrand B, Svensson H, Goldschimidt H, Ljungman P, Apperley $\mathrm{J}$, Mandelli F, et al. Alpha-interferon maintenance treatment is associated with improved survival after high-dose treatment and autologous stem cell transplantation in patients with multiple myeloma: a retrospective registry study from the European Group for Blood and Marrow Transplantation (EBMT). Bone Marrow transplantation 2001;27:511-515.

9. Ludwig H, Cohen AM, Polliack A, Huber H, Nachbaur D, Senn HJ, et al. Interferon-alpha induction and maintenance in multiple myeloma: results of two multicenter trials and summary of others studies. Annals of Oncology 1995;6:467-476.

10. Peest D, Deicher H, Coldewy R, Schmoll H, Schedel I. Induction and maintenance therapy in multiple myeloma: a multicenter trial of MP versus VCMP. Eur J Cancer Clin Oncol 1988; 24:1.061-1.067.

11. Salmon SE, Crowley JJ, Grogan TM, Finley P, Pugh RP, Barlogie B. Combination chemotherapy, glucocorticoids, and interferon alfa in the treatment of multiple myeloma: a Southwest Oncology Group Study. Journal of Clinical Oncology 994; 12:2.405-2.414.

12. Zervas K, Pouli A, Perifanis V, Papanastasiou K, Chatziyianni M, Mitsouli C, et al. Maintenance therapy with interferon-alpha(IFNalpha) versus IFN-alpha plus chemotherapy in multiple myeloma (MM) The Greek Myeloma Study Group. Eur J Haematol 1996; 57:142-148.

13. Wisloff F, Andersen P, Andersson TR, Brandt E, Eika C, Fjaestad $\mathrm{K}$, et al. Incidence and follow-up of asymptomatic multiple myeloma. The myeloma project of health region I in Norway. European Journal of Haematology 1991;47:338-341.

14. The Myeloma Triallist's Collaborative Group. Interferon as therapy for multiple myeloma: an individual patient data overview of 24 randomized trials and 4.012 patients. Br J Haematol 2001;113: 1.020-1.034.

15. Barlogie B, Kyle R, Anderson K, Greipp P, Lazarus H, Jacobson J et al. Comparable survival in multiple myeloma (MM) with high dose therapy (HDT) employing MEL $140 \mathrm{mg} / \mathrm{m}^{2}+\mathrm{TBI} 12 \mathrm{GY}$ autologous versus standard dose therapy with VBMCP and no benefit from interferon (IFN) maintenance: results of Intergroup Trial S9321. Blood 2003;102: 42A.

16. Crowley JJ, Fonseca R, Greipp P, Mccoy J. Comparable survival in newly diagnosed multiple myeloma (MM) after VAD Induction with high dose therapy using Melphalan $140 \mathrm{mg} / \mathrm{m}^{2}+\mathrm{TBI} 12 \mathrm{GY}$ (MEL + TBI) versus standard therapy with VBMCP and no benefit from interferon (IFN) maintenance: final results of Intergroup Trial S9321 in the context of IFM 90 and MRV VII trials. Blood 2004;104:156a.

17. Schaar CG, Kluin-Nelemans HC, Marvelde C, Cessie S, Breed WP, Fibbe WE, et al. On behalf of the Dutch-Belgian Hemato-Oncology Cooperative Group HOVON. Interferon- $\alpha$ as maintenance therapy in patients with multiple myeloma. Annals of Oncology 2005; 16:634-639.

18. Mahendra P, Hood J, Johnson D, Ethell M, Jestice H, Scott M et al. Autografting for multiple myeloma: a 5-year experience at a single institution. Bone Marrow Transplant 1995;16:759-763.

19. Bensinger W, Rowley S, Demirer T, Lilleby K, Schiffman K, Clift $\mathrm{R}$, et al. High-dose therapy followed by autologous hematopoietic stem-cell infusion for patients with multiple myeloma. J Clin Oncol 1996;14:1.447-1.456.

20. Barlogie B, Jagannath S, Desikan K, MattoX S, Vesole D, Siegel D, et al. Total therapy with tandem transplants for newly diagnosed multiple myeloma. Blood 1999;93:55-65.

21. Greenstein S, Ghias K, Krett N, Rosen S. Mechanisms of glucocorticoid-mediated apoptosis in hematological malignancies. Clinical Cancer Research 2002;8:1.681-1.694. 
22. Palumbo A, Boccadoro M, Garino LA, Gallone G, Frieri R, Pileri A. Multiple myeloma: intensified maitenance therapy with recombinat interferon-alpha-2b plus glucocorticoids. Eur Journal Haematol 1992;2:93-97.

23. Alexanian R, Weber D, Dimopoulos M, Delasalle K, Smith TL. Randomized trial of alpha-interferon or dexametasone as maintenance treatment for multiple myeloma. Am Journal Hematol 2000;65:204-209.

24. Shustick C, Belch A, Robison S, Rubin S, Dolan S, Kovacs M et al. Dexamethasone (dex) maintenance versus observation (obs in patients with previously untreated multiple myeloma: a National Cancer Institute of Canada Clinical Trials Group study: MY7. Proc Amer Soc Clin Oncol 2004;23:558.

25. Berenson J, Crowley JJ, Grogan TM, Zangmeister J, Briggs A, Mills $\mathrm{G}$, et al. Maintenance therapy with alternate-day prednisone improves survival in multiple myeloma patients. Blood 2002;99: 3.163-3.168.

26. Salmon SE, Crowley JJ, Balcerzak SP, Roach RW, Taylor SA, Rivkin SE, et al. Interferon versus interferon plus prednisone remission maintenance therapy for multiple myeloma: a Southwest Oncology Group Study. Journal clinical Oncology 1998; 16:890-896.

27. Stewart KA, Chen C, Howson-Jan K, White D, Roy J, Kowacs M, et al. Results of a multicenter randomized phase II trial of thalidomide and prednisone maintenance therapy for multiple myeloma after autologous stem cell transplant. Clinical Cancer Research 2004; 10:8.170-8.176.

28. Rajkumar V, Fonseca R, Dispenzieri A, Lacy M, Lust J, Witzig T, et al. Thalidomide in the treatment of relapsed multiple myeloma. Mayo Clin Proc 2000;75:897-901.

29. Feyler S, Jackson G, Rawstron A, El-Sheriny Y, Snowden J, Roderick J. Thalidomide maintenance following high dose therapy in multiple myeloma: A UK Myeloma Forum Phase 2 Study. Blood 2003; 102:2558 abst.

30. Sahebi F, Spielberger R, Kogut NM, Fung H, Falk PM, Parker P, et al. Maintenance thalidomide following single cycle autologous peripheral blood stem cell transplant in patients with multiple myeloma. Bone Marrow Transplantation 2006;37:825-829.

31. Santos ES, Goodman M, Byrness JJ, Fernandez HF. Thalidomide efects in the post-transplantation setting in patients with multiple myeloma. Hematology 2004;9:35-39.

32. Attal M, Harousseau JL, Leyraz S, et al. Maintenance treatment with thalidomide after autologous transplantation for myeloma: first analysis of a prospective randomized study of the Intergroupe Francophone du Myeloma (IFM 99 02). Proceeding of the American Society of Hematology. Blood 2004;104:155a abst 535

33. Attal M, Harousseau JL, Leyraz S, et al. Maintenance therapy with thalidomide improves survival in multiple myeloma patients. Blood 2006;108(10):3.289-3.294.

34. Brinker BT, Waller EK, Leong T, Heffner LT, Redei I, Langston AA, et al. Maintenance therapy with thalidomide improves overall survival after autologous hematopoietic progenitor cell transplantation for multiple myeloma. Cancer 2006;15:2.171-2.180.

35. Barlogie B, Tricot G, Anaissie E, Shaughnessy J, Rasmussen E van rhee F, Fassas A, et al. Thalidomide and hematopoietic cell transplantation for multiple myeloma. NEJM 2006;354 (10): 1.076-1.078.

36. Diaz C, Soutelo MJ, Quiroga L, Palmer L, Lutfi R. Tratamiento del mieloma multiple com pamidronato endovenoso. Mejoria del dolor y supresion del riesgo de hipercalcemia. Medicina 2004; 64:289-294.

37. Kondo H, Mori A, Kubota M. Maintenance with pamidronate following first-line MP or VAD therapy in multiple myeloma. Leukemia \& Lymphoma 2003;44:303-307.
38. Berenson J, Lichtenstein A, Porter L, et al. Longterm pamidronate treatment of advanced multiple myeloma patients reduces skeletal events. Myeloma Aredia Study Group. J Clin Oncol 1998;16:513-602.

39.Burie B. New approaches to treatment for multiple myeloma: durable remission and quality of life as primary goals. Clin Lymphoma Myeloma 2005;6:181-190.

40. Coscia M, Mariani S, Battaglio S, Di Bello C, Fiore F, Foglietta M, et al. Long-term follow-up of idiotype vaccination in human myeloma as a maintenance therapy after high-dose chemotherapy. Leukemia 2004;18:139-145.

41. Kwak LW, Tawb DD, Duffey F, et al. Transfer of myeloma idiotypespecif immunity from actively immunized marrow donor. Lancet 1995;345:1.016-1.020.

42. Richardson PGG, Barlogie B, Berenson J, Singhal S, Jagannath S, Irwin $\mathrm{D}$, et al. Clinical factors predictive of outcome with bortozomib in patients with relapsed, refractory multiple myeloma. Blood 2005;106(9):2.977-2.981.

43. Knop S, Hebart H, Kunzmann V, Angermund R, Einsele H. Bortozomib once weekly is well tolerated as maintenance therapy after less than a complete response to high-dose Melphalan in patients with Multiple Myeloma. Blood 2006;108(11). Abstract \#5099.

O tema apresentado e o convite ao(s) autor(es) consta da pauta elaborada pelo co-editor.

Avaliação: Co-editor e um revisor externo. Publicado após revisão e concordância do editor. Conflito de interesse: não declarado.

Recebido: 25/11/2006

Aceito::15/01/2007 